\title{
Design and Implementation of Online Shopping System Based on the Struts Framework
}

\author{
Zhang $\mathrm{Li}^{1, *}$ and Zhao Luhua ${ }^{2}$ \\ ${ }^{1}$ College of Computer Engineering, Jiangsu University of Technology, ChangZhou, China \\ ${ }^{2}$ Modern Education Technology Research Center, Henan Quality Polytechnic, Pingdingshan, China
}

\begin{abstract}
Struts is currently a prevalent Web application development framework based on MVC. It has prominence in the open, large and extensible J2EE-based Web application development. Through combining the actual operation process of E-commerce enterprise with the developed requirement, this paper categorically analyses MVC actual implementation technology, analyses and describes the Struts framework. Finally it gives a demonstrative example of Category management module, which explains in detail technical implementation to E-Online Shopping System based Struts framework and presents function description of correlative constitution section. By practical application, it shows the system improves platform's security and stability.
\end{abstract}

Keywords: Controller component, Model component, MVC pattern, Online shopping system, Struts framework.

\section{INTRODUCTION}

With the development of internet technology, network service plays an increasingly important role in people's daily life. People expect that they can get the satisfied service or goods in a convenient way and in very short time. Hence, the electronic commerce system at this moment plays a very critical part. On one hand, it is very convenient for people to look at the goods online and it also shortens people's time period for shopping. On the other hand, for the enterprise, it shortens intermediate links, and it can reduce the geographic restrictions and decreases the merchandise inventory pressure, therefore, it can greatly save business operating cost. However, this system also faces problems that the form of ecommerce is very changeable; as a result, the inner structural can be complex and varied. Then, how to deal with that? Based on the development model of Struts [1], it can effectively deal with these varied requirements. It completely changes the previous traditional J2EE development mode that is the coupling of display, control and business which makes software reuse difficult, cooperation and division of the team difficult, development time long and cost high.

Therefore, this article based on the integrated development environment of MyEclipse and using MySQL as the backend database, the MVC OF Struts designed a crossplatform, extensible $\mathrm{B} / \mathrm{S}$ electronic commerce system. This system is runs on the internet where users can browse the goods and buy them freely, they can even choose the payment method. This system innovates the traditional shopping, which can make people buy what they want at home.
The computational results of the system states that the system operates well and that its security and expandability are also high.

\section{INTRODUCTION OF STRUTS}

Struts [2] is an exceptional MVC frame. It combines features of Servelet and JSP and inherits various features of MVC, and it changes and extends according to the J2EE'S characteristic. There are three main parts of Struts:

The controller is responsible for the Action that is processed by a specific operation; JSP page (view); that applies business logic packaging. The master controller of Struts (ActionServlet) receives the request from client and according to the allocated route of the system (Struts-config. xml) HTTP requests the objects to the other Action. In these action objects, it will make business operation and after operation, it will transfer from ActionServlet to JSP, and the processed results will be returned to clients. The packaging data through Action Form can be mutual used in Model and View. The working principle of Struts is showing in Fig. (1).

\section{THE ANALYSIS AND DESIGN OF ELECTRONIC COMMERCE SYSTEM}

\subsection{The Demand Analisis and Case Design of the System}

The aim of this electronic commerce system is realized by the construction of this system. The most important thing is to attract customers to know the main product of the website, for instance an e-commerce website which mainly sells children's shoes or children's clothes and so on. For transactions, it has to build $\mathrm{B}$ to $\mathrm{C}$ sales model which are consumer 


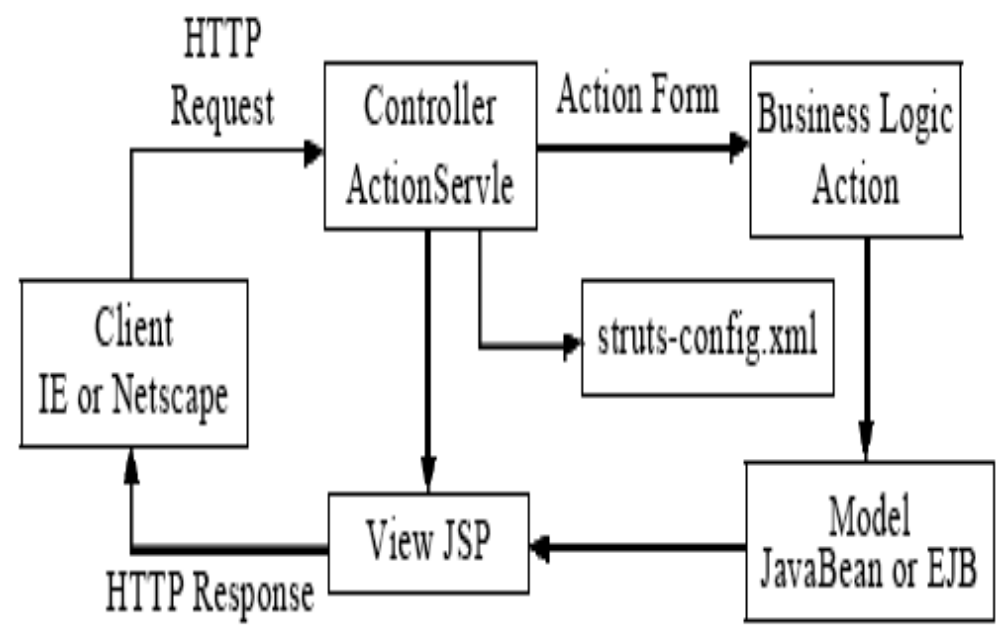

Fig. (1). Working principle of Struts.

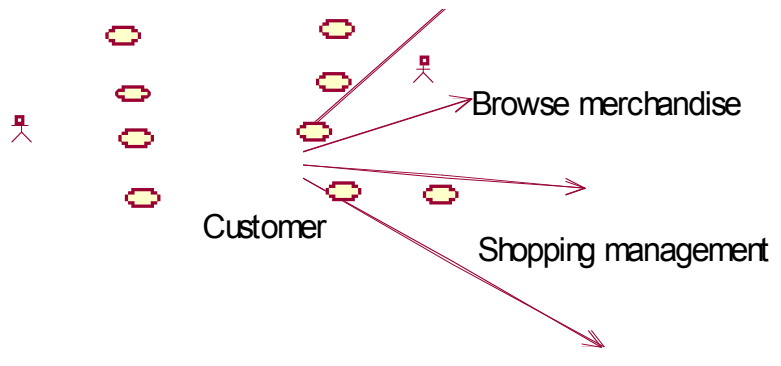

Commodity information feedback

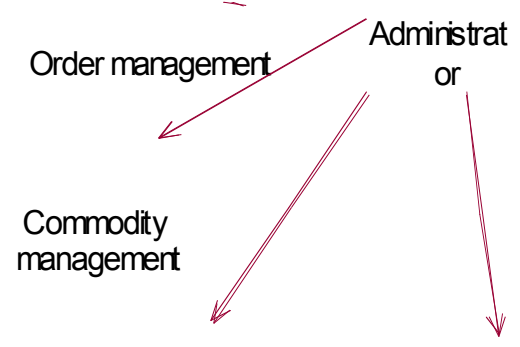

Commodity information release
User analysis and decision making

Fig. (2). The use case of e-shopping system.

facing, and it has to realize the integration of products, online payment, and logistic services. Moreover, it needs to make a customized search engine and data analysis system to find the potential and key customers. It also needs to analyze the market trend to build a scientific decision system [3].

Therefore, the system requirement description is as following: electronic commerce system includes two subsystems; one is for the front desk sale and display. When clients $\log$ in, they can freely look over and search their favorite products (for example: off-price goods, new arrivals). Meanwhile, customers can put the products they like in a "shopping cart" and which they can later modify, (add or delete), then they can submit the order form to service counter to pay. The other subsystem is in the backstage management system. Merchants can manage the relevant information published, typed-in and alteration. It can also manage the search of the order and manage the registered clients of on-line sales system. Meanwhile, it can analyze consumer behavior, which can provide evidence for better scientific decision for the corporation. Fig. (2) is the use case of this eshopping system.

\subsection{The E-R Design of Online Shopping System}

PowerDesigner [3, 4] is the CASE tool set of Sybase, it can make data flowchart, concept data model, physical data model and can also control the generated data model. This system uses PowerDesigner to set up data and induct E-R model to MySQL, and generate physical data sheets. Therefore, according to the above description, combined with the need and description of 2.1, the design of E-R can be finished. However, there are numerous system database sheets, only some important database sheets are listed here. The relationship among them will be explained here. The specific design of E-R is in (Fig. 3).

\subsection{Structure Design of Online Shopping System}

In this article, the system uses Struts based on MVC, which makes great convenience for the developer to make modularization exploitation. It greatly increased code's reusability and maintainability, and it also takes the crossplatform of this system into consideration. Fig. (4) shows the design model of this online shopping system.

(1) View is responsible for the systems visual theme and realization, and View is composed by JSP and Action Form bean. JSP contains static HTML, CSS, Div and Struts label database. Action Form bean is responsible for the data transmission between JSP and Model.

(2) Controller is composed by Action Servlet. Its task are: first, it has to finish all the initialized work, that is to read the 


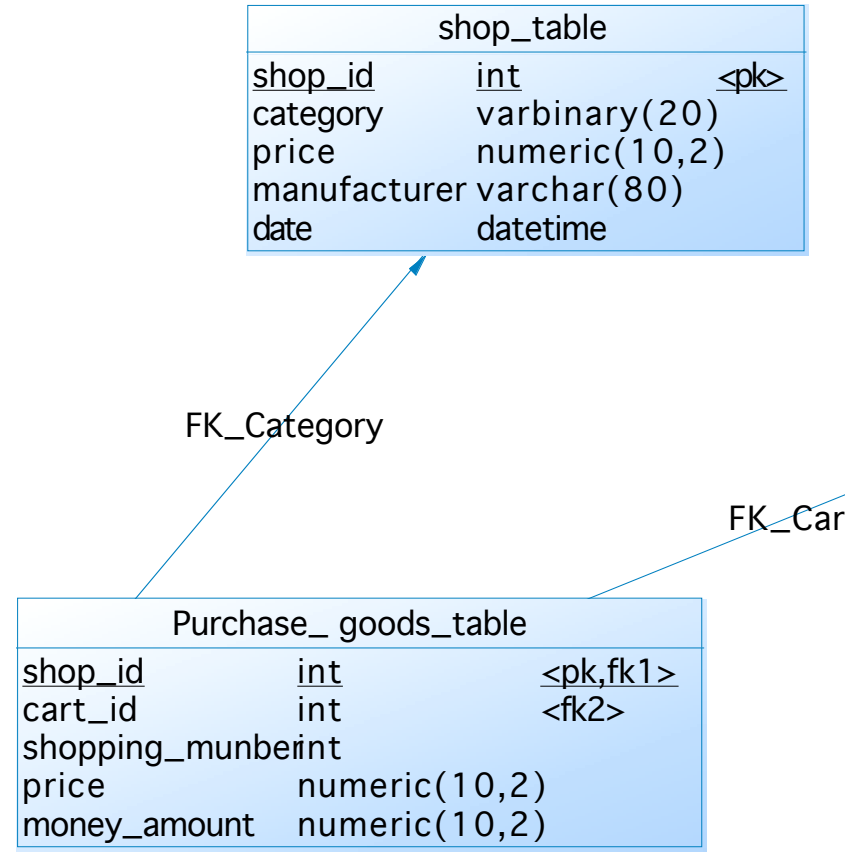

Fig. (3). The E-R Design of electronic commerce system.

information in Struts-config. xml and intercept the matching map of URL by Action Servlet; second, according to the intercepting request initiate Action Form bean; last, to find the corresponding Action subclass in Action Mapping: if there are no corresponding Action, then transmit the request to JSP; if there are corresponding Action, then initiate ActionForm bean and use HTTP to fill the data and its property, and save these results in request for other Action or JSP to use.

(3) in the Model [4], the specific business logic operation is finished by Action. In order to reduce the coupling relationship between business logic and database manipulation, the DAO is used to separate business logic and data access. It can increase the flexibility and maintainability of system for that DAO model Action [5] and provides abstract data access port. Therefore, Model doesn't need to care about selection, insertion, deletion, and updating, hence, it avoids mixed call statement in service code, so the business practice can be much clearer and on the other hand, because of the separation of data access port and data access, which can make the developer concentrate on business logic code rather than data's selection, insertion, deletion, and check.

\section{THE IMPLEMENTATION OF ELECTRONIC COMMERCE SYSTEM BASED ON STRUTS}

Struts applied MVC design model, which separated page display, data control and business operation makes the exploitation and maintenance more convenient and reasonable. For online shopping electronic commerce system $[6,7]$, the construction methods of all modules are basically same. Next set commodity management module [8] as an example to explain the develop process of online shopping electronic commerce system based on Struts.

\subsection{Design of View Module}

The main function of View is to display data to users, and in this system, View is composed by Struts, Div, CSS, HTML, JSP and formbean. The advantage of this is that it can avoid the coupling of Java source code and HTML statement in JSP, and it also can enhance reusability of View. It can simplify the page development, and is good for test and maintenance..

First, use Dreamweaver to establish JSP: 1), add and modify Category page addCategory.jsp ; 2), delete some category page delCategory.jsp ; 3 ), search category page searchCategory.jsp; 4), maintain category information page categoryInfoMaint.jsp. It can display all the products information and it can add, delete, modify and edit the selected products.

Lastly, to define and describe the CategoryFormbean. It inherited the org.apache.struts.action.ActionForm in Struts. The main function of this class is to collect and display data. The goal of data collection is to provide a business model for Model module. And display data is to show the conducted data to users. The specific steps are:

First: introduce ActionForm to Struts;

Second. write down the specific codes as following:

public class MerForm extends ValidatorForm

\{

// 1.define products manufacturer

private String manufacturer;

//2. define products price

private Double sprice; 


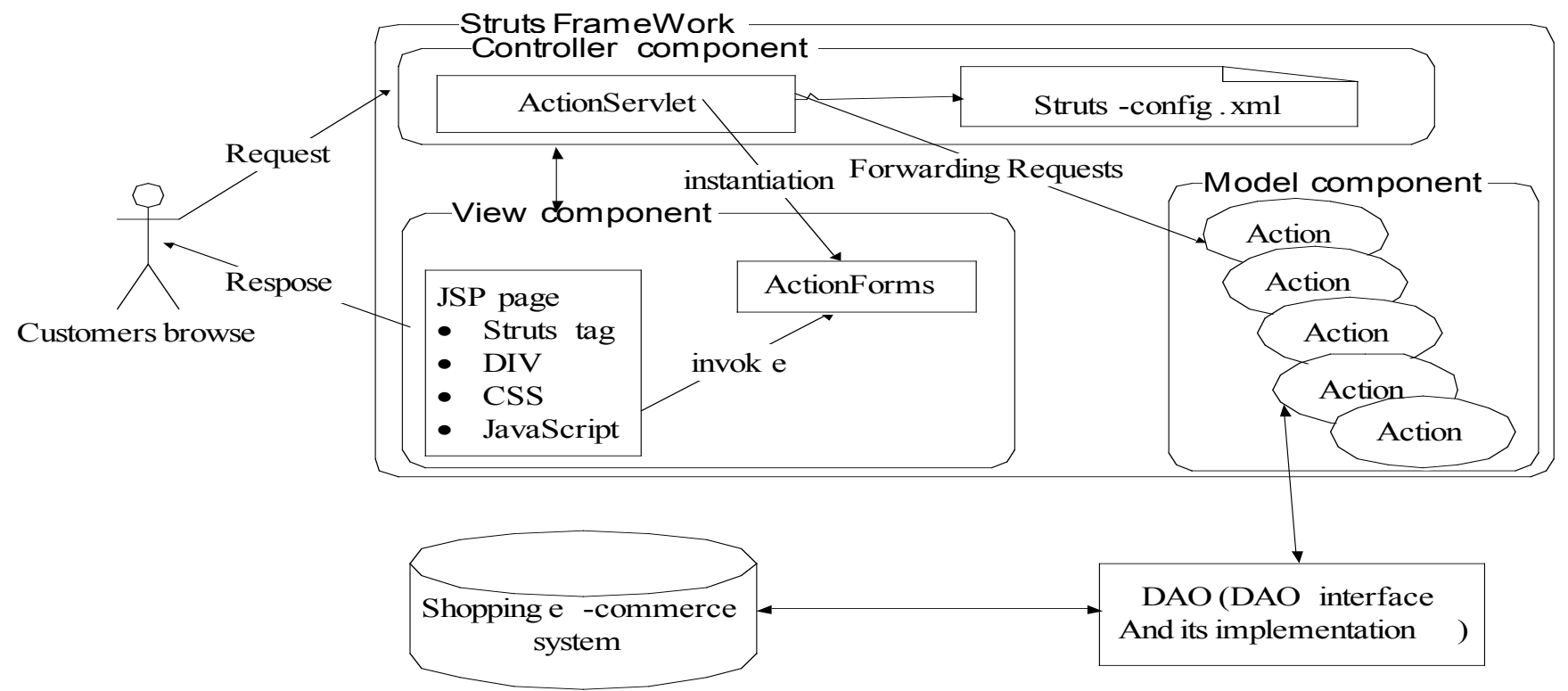

Fig. (4). The framework of online shopping electronic commerce system based on Struts.

// 3.define products discount

private Integer special;

/omit other property, meanwhile,

//generate corresponding Getter/Setter

\}

\subsection{Implementation and Design of Controller}

Controller is the core of all operations, whenever, ActionServlet of controller deals with all the things, it decides all the flow relation of every module in this system. Then how to finish these works? Firstly, it is necessary to register ActionServlet and Struts-config.xml in Web.xml. Web.xml is the place where Controller's modules ActionServlet and struts-config.xml are described and among these, *.do stands for requesting mapping, the specific allocations are:

$<$ servlet $>$

$<$ servlet-name $>$

action

$</$ servlet-name $>$

$<$ servlet-class $>$

org.apache.struts.action.ActionServlet

$</$ servlet-class $>$

$<$ init-param $>$

$<$ param-name $>$ config $<$ /param-name $>$

$<$ param-value $>$

/WEB-INF/struts-config.xml
$</$ param-value $>$

$<$ init-param $>$

$</$ servlet $>$

$<$ servlet-mapping $>$

$<$ servlet-name $>$ action $</$ servlet-name $>$

$<$ url-pattern $>*$.do $</$ url-pattern $>$

$<$ /servlet-mapping $>$

$<$ servlet $>$

Secondly, to collocate struts-config.xml, in strutsconfig.xml, its task is to ensure the navigation relationship of all the pages, and its relationship is finished by ActionMapping, Action and ActionForm. Its advantages are: it is easy to be developed, the cost of maintenance is low, and process is clear. Parts of the allocations are:

$<$ action attribute=" MerForm" input="/reg.jsp"

name $=$ " merForm" path $=" / \mathrm{mem} "$

scope $=$ "request"

type $=$ "com.struts.action.MemAction">

$<$ forward name="browseWord"

path="/searchCategory.jsp"/>

$<$ forward name="loadMember"

path="/categoryInfoMaint.jsp"/>

$</$ action $>$

\subsection{Implementation and Design of Model}

In this module, the main design of Model [9] is the design of Action, and here, ActionForm is used as request's parameter by ActionServlet introduced for corresponding 
Action. Detailed process can be seen in 3.2.2. so it is responsible for the description of business logic and data processing. According to (Fig. 4), data processing is finished by DAO. Therefore, in Action, it is no need to consider about pages, the developing of procedure can be done under Java. The specific development steps of MemAction are as following:

Firstly, to design the DAO port:

public interface MerService \{

$/ * *$ browse productscategory */

public List browseCategory() throws Exception;

\}

Secondly, to finish the implementation of DAO data processing:

public List browseCategory() \{

Session session $=$ MySessionFactory.getSession();

Transaction $\mathrm{tx}=$ null;

List list $=$ null;

$\operatorname{try}\{$

Query query = session.createQuery("from Category as a order by a.id");

$\mathrm{tx}=$ session.beginTransaction( $)$;

list $=$ query.list () ;

tx.commit();

if (!Hibernate.isInitialized(list))

\{

Hibernate.initialize(list);

\}

$\operatorname{catch}($ Exception ex)

\{

if(tx!=null)tx.rollback();

logger.info("when carry out browseCategory in MerServiceImp, the method is wrong $\backslash n "$ );

ex.printStackTrace();

\}

Finally

\{

MySessionFactory.closeSession();

\}

return list;

\}

Lastly, to undertake business logic in MemAction. public classMemActionextends Action \{

the specific arithmetic is as following:

//1 obtain the data from view

// 2 conduct data processing

$/ / 3$ processing business logic and data

//4 implement the skip of view pages

\}

The above codes gets the data from view by merForm and then use merForm to finish data processing. The last line of codes gives the view of steering "success", this view is allocated in the codes of III.B. If the flow wants to be changed, it only needs to modify Struts-config.xml.

\section{RELEASE SYSTEM}

This system uses Tomcat server, when the development of this system is finished, it can be packed as a binary file of war through Myeclipse, and it can be copied to webapps in Tomcat server. Then this system can be operated.

\section{CONCLUSION}

This system covered products scanning, shopping cart management, user's registration, logging, etc. It passed the performance testing, it is stable. Practice has proved; using Struts, divides the work to page designing, process control, model design and implementation, makes the system much clearer on structure. It can reduce the coupling of each part and simplify the efficiency of software development. Meanwhile, it increased the performance and reduced maintenance of the system and it embodies the J2EE technology.

\section{CONFLICT OF INTEREST}

The authors confirms that this article content has no conflict of interest.

\section{ACKNOWLEDGEMENTS}

This study is supported by the Science and Technology Development Planning Project of Shandong Province (No. 2013YD05004), People's Republic of China.

\section{REFERENCES}

[1] Z. Li, "Application of MVC pattern in data middleware", Computer Engineering, vol. 36, pp. 70-72, 2010.

[2] Z. Li, and Z.W. Xi, "Design and realization persistence framework of tourism e-business system", Journal of Wuhan University of technology, vol. 32, pp. 113-117, 2010.

[3] Z. Li, and Z.W. Xi, "Design and implementation of fixed assets management system based on JavaEE" Computer Engineering and Design, vol. 30, pp. 3797-3800, 2009.

[4] Z. Li, "Design and realization of personal internet banking system based on multi-tier architect" Journal of Applied Sciences, vol. 13, pp. 4386-4392, 2013.

[5] S.J. Metsker, and W.C. Wake, Design Patterns in Java. America: Addison Wesley, 2009. 
[6] Z. Li, "Design and realization of car rental managerment system based on AJAX+SSH", Information Technology Journal, vol. 12, pp. 2756-2761, 2013.

[7] E. Gamma, R. Helm, R. Johnson, and J. Vlissides, Design Patterns: Elements of Reusable Object-Oriented Software, America: Addison Wesley/Pearson, 2009
[8] J. Hong-Chao, Y. Yi-Xin, and B. X-Juan, "Struts framework application based on SOA", Computer Engineering, vol. 35, no. 1, pp. 21-23, 2009

[9] M. Young, The Technical Writer's Handbook. Mill Valley, CA: University Science, 1989.

Received: September 22, 2014

Revised: November 05, 2014

Accepted: November 06, 2014

(C) Li and Luhua; Licensee Bentham Open.

This is an open access article licensed under the terms of the Creative Commons Attribution Non-Commercial License (http://creativecommons.org/licenses/by$\mathrm{nc} / 3.0 /$ ) which permits unrestricted, non-commercial use, distribution and reproduction in any medium, provided the work is properly cited. 